\title{
Chapter 12 \\ Relevance of Individual-Level Residential \\ Outcomes for Describing Segregation
}

The new options for segregation analysis introduced here suggest a new basis for evaluating segregation indices - the substantive relevance of the individual-level residential outcomes registered by the indices. Three of the segregation indices considered here - G, D, and S - have been used widely in empirical analyses for more than five decades and each has been reviewed many times in methodological studies. ${ }^{1}$ Until now little attention has been given to the substantive relevance of the individual-level residential outcomes each index registers. In this chapter I argue that it is instructive to consider how indices differ on this important point of comparison.

In their difference of means formulations $\mathrm{G}, \mathrm{D}$, and $\mathrm{S}$ register group differences on average residential outcomes (y) scored from (pairwise) area group proportion (p). $\mathrm{G}$ rescales $\mathrm{p}$ to register relative rank or percentile scoring. $\mathrm{D}$ rescales rank distinctions on $\mathrm{p}$ to register only a 0,1 coding of whether or not $\mathrm{p}$ is above the city average $(\mathrm{P})$. $\mathrm{S}$ does not rescale $\mathrm{p}$; it registers it in its original metric. Because $\mathrm{S}$ registers $\mathrm{p}$ directly, a given value of $\mathrm{p}$ yields the same value of $\mathrm{y}$ in all cities. In contrast, $\mathrm{G}$ and $\mathrm{D}$ assign values of $\mathrm{y}$ based on monotonic, rank position scoring schemes that vary in functional form in complex ways across cities. In particular, the scoring of $y$ from $\mathrm{p}$ is nonlinear and the magnitude of the departure from linearity varies with city racial mix (as discussed previously in Chap. 5). Consequently, identical values of area racial proportion (p) can be and often are assigned very different values on the residential outcome of scaled contact (y) in different cities.

Residential outcomes (y) registered by $\mathrm{S}-$ for example, area proportion White (p) in the familiar case of White-Black segregation - have clear substantive appeal. The residential outcome of group contact in its "natural" metric is directly meaningful to individuals and households both in its own right and also because area proportion White $(\mathrm{p})$ tends to correlate with neighborhood characteristics that have clear relevance for life chances (e.g., crime rates, quality of schools, neighborhood services and amenities, property values, etc.). The same cannot be said for the

${ }^{1} \mathrm{I}$ bring $\mathrm{R}$ and $\mathrm{H}$ into the discussion later in this chapter. 
neighborhood outcomes (y) used in computing scores for G and D. G rescales values of $\mathrm{p}$ into ordinal-level, relative rank (percentile) scores. D collapses values of $\mathrm{p}$ to just two relative rank scores.

The value and sociological relevance of scoring residential outcomes (y) as $G$ and D do is not obvious. Few if any discussions of group differences in residential outcomes explicitly prioritize ordinal position on contact with Whites over contact with Whites in its natural metric. Similarly, discussions that view area proportion Whites as relevant for the impact of area of residence on life chances rarely if ever suggest that this is best captured by coding area proportion White in terms of relative rank position or in terms of "parity." To the contrary, theories of majority group discrimination and avoidance of minority groups usually presume that exclusionary discrimination by Whites and White avoidance of minority areas is aimed at maintaining neighborhoods as predominantly-majority (e.g., $85 \%$ White or higher) rather than simply "above parity" in comparison to proportion White in the city which of course can vary dramatically across cities. In view of this, I believe there is no compelling basis for giving "relative rank" scoring of p or "above parity" scoring of $\mathrm{p}$ priority over the natural interval metric for $\mathrm{p}$.

$\mathrm{S}$ also is attractive because it has clear, straightforward implications that are easy to explain to general audiences. For example, if White-Black segregation as measured by $S$ is high - say 60 - it means Blacks' average contact with Whites is 60 points lower than Whites' average contact with Whites. This yields an unambiguous signal about the consequences of segregation for individuals and groups; it indicates that, on average, Whites live in predominantly White neighborhoods and Blacks live in predominantly Black neighborhoods. This score on $\mathrm{S}$ also sends a signal about the extent to which Whites and Blacks can potentially experience differences on life chances based on neighborhood characteristics that correlate with area proportion White. When $\mathrm{S}$ is zero, Whites and Blacks will necessarily experience the same average on all residential outcomes. As $\mathrm{S}$ increases above zero, so too does the potential for Whites and Blacks to experience differences on other important residential outcomes (e.g., crime, poverty, schools, amenities, etc.).

The simple and clear conclusions one can draw based on knowing that $\mathrm{S}$ takes a high score do not necessarily hold when $\mathrm{G}$ and $\mathrm{D}$ take high scores. To the contrary, as discussed in Chaps. 7 and 8, it is possible for $G$ and $D$ to be very high - say $80-$ and for both Whites and Blacks to live in neighborhoods that on average are similar on area proportion White (p). In these cases it could be highly misleading to assume that high scores on $\mathrm{G}$ and $\mathrm{D}$ carry consequences for group differences on neighborhood outcomes that are relevant for life chances (e.g., crime, poverty, schools, amenities, etc.) and are correlated with area proportion White. The reason for this is simple. If Whites and Blacks experience similar outcomes on area proportion White, they will, all else equal, tend to experience similar outcomes on factors that are correlated with area proportion White.

The mathematical basis for how $\mathrm{G}$ and $\mathrm{D}$ can take high values when Whites and Blacks share similar neighborhood outcomes was discussed earlier in Chap. 5. It was illustrated in the graphs in Fig. 5.1 which depict how G and D register group differences in contact with Whites (p) after $\mathrm{p}$ has been subjected to a dramatic 
nonlinear rescaling. This nonlinear rescaling of $p$ reduces the importance of group differences on contact with Whites ( $p$ ) over some ranges of $p$ and it exaggerates the importance of group differences on $\mathrm{p}$ over other ranges of $\mathrm{p}$. In the graph for the White-Asian comparison in Fig. 5.1, for example, group differences in p over the range of $0-80$ are of minor importance while group differences on $p$ over the range 80-100 take on great importance. $D$ is even more extreme in this regard; it rescales values of $\mathrm{p}$ into values of $\mathrm{y}$ based on a one-step function that registers only differences on either side of P. The discussion in Chap. 7 further outlined the technical basis for how these characteristics of $\mathrm{G}$ and $\mathrm{D}$ create the possibility that they can and often will take values that differ substantially from values of S.

The key implication is that high values on $\mathrm{G}$ and $\mathrm{D}$ have uncertain implications for group differences on sociologically meaningful residential outcomes because values of $\mathrm{D}$ and $\mathrm{G}$ can be highly sensitive to small differences in area group proportion. Specifically, G and D for White-Black segregation can in principle take very high values when Whites and Blacks live in neighborhoods that, on average, are fundamentally similar on area proportion White (p) and other sociologically important neighborhood outcomes.

This may be surprising to some. If so, it is instructive to carefully consider the familiar interpretation of $\mathrm{D}$ as indicating the minimum proportion of one group that must move to bring about even distribution. Note that this interpretation implies nothing specific about whether the residential movement that eliminates uneven distribution as measured by $\mathrm{D}$ will cause either group's residential outcomes to change in sociologically important ways. In fact, the movement associated with eliminating a high value for $\mathrm{D}$ can and sometimes will produce small, potentially trivial, average changes in substantively meaningful residential outcomes for the members of a group.

This frames a point of clear contrast between $\mathrm{G}$ and $\mathrm{D}$ on the one hand and $\mathrm{S}$ on the other. High values of S always signal that residential movement needed to bring about even distribution will produce dramatic changes in group differences in residential outcomes. This is not necessarily true for $G$ and $D$. This is a consequence of the fact that high values of G and D can occur under "prototypical segregation," which involves high levels of group separation, but also under "dispersed displacement" or "displacement without separation" as discussed in Chap. 7 (Fig. 7.1).

The potential for $\mathrm{G}$ and $\mathrm{D}$ to manifest this characteristic is not uniform across all circumstances. It varies dramatically with relative group size. The underlying technical basis for this was reviewed in Chap. 7 and the logically possible consequences for D-S differences were summarized graphically in Figs. 7.4 and 7.5. The implications for empirical analyses also were illustrated in the comparison of the function $y=f(p)$ for $G / 2$ in the graphs in Fig. 5.1. The graph for the White-Latino comparison has the mildest nonlinearity in the scoring of $y$ from $\mathrm{p}$ because it has the most balanced group ratio of 68/32. In contrast, the group ratio of $92 / 8$ for the WhiteAsian comparison is much more imbalanced and the nonlinearity is much more pronounced in the graph for this comparison. The White-Black comparison is in between on both the group ratio of $76 / 24$ and the nonlinearity of the $y-p$ relationship. 

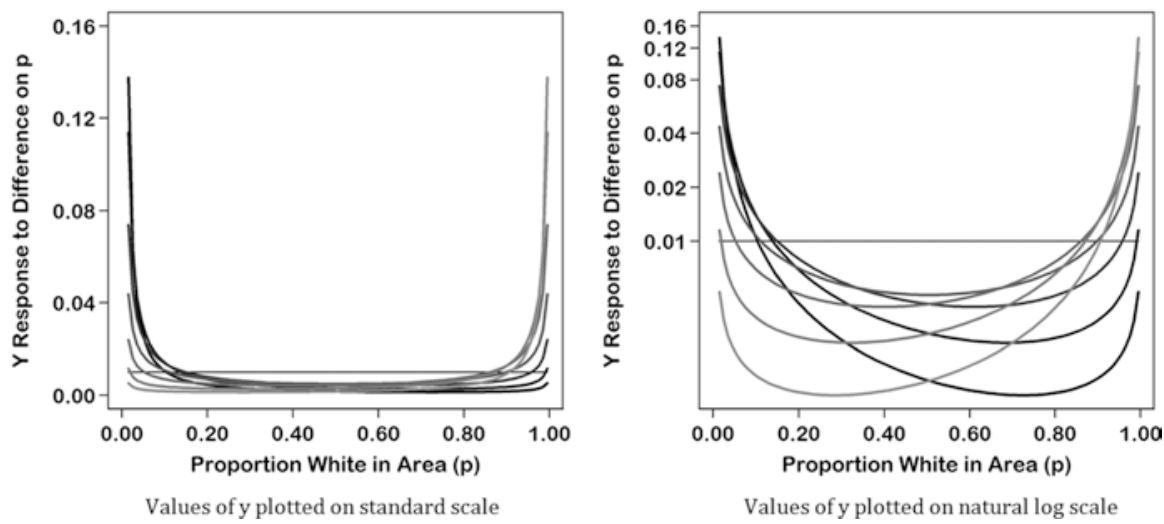

Fig. 12.1 Response of group contact (y) scored for Hutchens R by proportion White in area (p) and selected values for city proportion White (P). Curves reflect the response of Hutchens' $\mathrm{R}$ to a change in area proportion White (p) by level of $\mathrm{p}$ and selected values of proportion White for the city $(\mathrm{P}) . \mathrm{y}=\mathrm{f}(\mathrm{p})=Q+(1-\sqrt{p q / P Q}) /(p / P-q / Q)$. Moving from darker curves to lighter curves, the values of $P$ are: $0.01,0.05,0.20,0.50,0.80,0.95$, and 0.99 . The horizontal line is for reference and reflects the "flat" response of the separation index (S)

Further insight into these patterns can be gained by again considering the behavior of the function $y=f(p)$ for the Hutchens square root index (R) shown earlier in Fig. 6.6. The $y-p$ relationship for $\mathrm{R}$ is continuous and thus lends itself more easily to mathematical and graphical analysis than the $y-p$ relationship for $G$ which is mathematically less tractable because it is based on a percentile transformation. In other key respects, however, $\mathrm{R}$ and $\mathrm{G}$ are quite similar: the $\mathrm{y}-\mathrm{p}$ relationships for both $\mathrm{R}$ and $\mathrm{G}$ have similar nonlinear forms (i.e., both follow a backwards $\mathrm{S}$ curve); the nonlinearity in the $y-p$ relationships for both $\mathrm{R}$ and $\mathrm{G}$ become more pronounced when group size is more imbalanced; and $\mathrm{R}$ and $\mathrm{G}$ are closely correlated in empirical data sets.

The graph in Fig. 6.6 plots the function $y=f(p)$ for $R$ over selected values of city racial mix $(P)$. The graph documents that $y=f(p)$ for $R$ is always a continuously rising backwards $S$ curve. The nonlinear nature of the $y-p$ relationship means that $\mathrm{R}$ responds to differences on $\mathrm{p}$ in a much different way than $\mathrm{S}$. S registers differences arithmetically according to p's original, "natural" metric. R responds more strongly to differences on $p$ over ranges of $p$ where the curve is relatively "steep" and less strongly to differences on $\mathrm{p}$ over ranges of $\mathrm{p}$ where the curve is relatively "flat". The graph also reveals that where steep and flat regions of the curve occur over the range of $\mathrm{p}$ is strongly conditioned by the racial mix of the city $(\mathrm{P})$. When the two groups are balanced, the $y-p$ curve for $\mathrm{R}$ is symmetric and differences between how $\mathrm{R}$ and $\mathrm{S}$ respond to $\mathrm{p}$ are modest. When group size is imbalanced, the $\mathrm{y}-\mathrm{p}$ curve for $\mathrm{R}$ becomes asymmetric and more profoundly nonlinear. Under these conditions, the differences between how $\mathrm{R}$ and $\mathrm{S}$ respond to $\mathrm{p}$ can be dramatic.

This is documented further in Fig. 12.1 which depicts graphically how changes in $\mathrm{p}$ are registered as changes in $\mathrm{y}$ as scored for $\mathrm{R}$. The graph on the left uses the 
original metric scoring of $\mathrm{y}$; the graph on the right uses a natural log scale on the $\mathrm{y}$ axis. These two graphs make it clear that $R$ responds more strongly to changes in $p$ near the extremes of $\mathrm{p}$ and this tendency becomes more asymmetric and more dramatic when the racial composition of the city departs from balance (i.e., 50/50). This establishes the mathematical basis for how and when R (and G and D) can take high values when $\mathrm{S}$ is low. Regarding the "how" part of the story, $R$ (and $G$ and $D$ ) can take high values when $S$ is low by responding dramatically to very small differences on $p$. Regarding the "when" part of the story, the potential for $\mathrm{R}$ to depart from $\mathrm{S}$ is greatest when the city racial mix $(\mathrm{P})$ is highly imbalanced.

It is clear from these results that $\mathrm{R}$ must be high when $\mathrm{S}$ is high, but $\mathrm{R}$ can be either high or low when $\mathrm{S}$ is low. As noted earlier, this also applies with equal force to $\mathrm{G}$ and $\mathrm{D}$. Thus, if $\mathrm{S}$ is high, $\mathrm{G}$ and $\mathrm{D}$ must be high, but when $\mathrm{S}$ is low $\mathrm{D}$ and $\mathrm{G}$ can be either high or low. This is consistent with results presented earlier in Figs. 8.1 and 8.2 which depicted graphs of plotting scores for D against scores for S (and vice versa) for White-Minority segregation comparisons for CBSAs in 1990, 2000, and 2010. It is readily evident here that when $\mathrm{S}$ is high, D also is high. But when $\mathrm{S}$ is low, values of D vary dramatically; sometimes they are low and sometimes they are high. This raises an obvious question, "When S is low and D (or G or R) is high, is there a compelling reason for assigning sociological importance to the high values of D (or G or R)?" I am not aware of a reason that is (or could be) grounded in the consequences segregation will have for sociologically important group differences in residential outcomes.

The one reason that comes to mind is grounded, not in consequences for group differences in residential outcomes, but more literally in "volume of movement" consequences of policies seeking to redress segregation. High values of D do imply that a large fraction of one group must change area of residence to bring about even distribution. That can be sociologically consequential in policy situations such as school desegregation where students are literally redistributed across schools. Historically, the consequence has been especially important for minority populations who have often disproportionately born the burden of bussing.

The sociological relevance of this volume of movement policy consequence cannot be denied. But its relevance for choosing segregation indices can be discounted for two reasons. The first is that it is "beside the point" because historically literal "volume of movement" policy implications of high values of D have almost always played out in contexts of "prototypical segregation" where values of S also are high. The driving concern behind the policy to redress segregation of course was that racial segregation adversely impacted life chances in education by creating group separation and unequal educational opportunities. The sociological import of $\mathrm{D}$ is fundamental and real; but it is beside the point for the issue under discussion because $\mathrm{S}$ captures the same concern and thus D does not identify a "life chances" implication that $\mathrm{S}$ misses.

The second reason is that the policy implications of a high value of $\mathrm{D}$ are much less likely to have practical consequences in situations where $\mathrm{D}$ is high and $\mathrm{S}$ is low. The basis for saying this is that policy concerns about reducing segregation usually are rooted in concerns about the impact of segregation on inequality in life chances. 
When $\mathrm{D}$ is high and $\mathrm{S}$ is low, groups live together and experience similar neighborhood outcomes. In these situations moving across neighborhoods to achieve exact even distribution will have limited impact on group differences in neighborhood outcomes. Thus, since policies to promote integration are unlikely to be pursued solely for the purpose of achieving exact even distribution without implications for life chances, the policy implications of D's volume of movement interpretation are unlikely to come into play in practice.

So we come back to the issue of why one would focus on values of D, or its technically superior "close cousins" $\mathrm{G}$ and $\mathrm{R}$, over values of $\mathrm{S}$. To argue that high values of $\mathrm{R}, \mathrm{G}$, and $\mathrm{D}$ are sociologically important when $\mathrm{S}$ is low, one must advocate two unusual views about the sociological relevance of residential outcomes.

First, one must view differences on $\mathrm{p}$ as both very important over certain narrow ranges of $\mathrm{p}$ and also much less important over the rest of the logical range of $\mathrm{p}$.

Second, one also must view it as desirable to amplify this differential evaluation of differences on $\mathrm{p}$ by greater amounts when a city's racial mix is imbalanced.

To the best of my knowledge, no segregation researcher has articulated a compelling basis for assessing group differences in residential outcomes in this manner. Measurement approaches of this sort are not used when group differences on other socioeconomic outcomes such as education, occupation, and income are studied. So it is not obvious why such an approach would be seen as attractive when studying group differences in residential attainments relating to area racial mix and group contact.

To be clear, I am not arguing that G, D, and R should not be used to measure uneven distribution. Researchers can be interested in uneven distribution for many different reasons. In some cases they may determine that one of these indices is the best choice to serve the needs of a particular study. As just noted, these measures might be defensible choices if one is interested in certain consequences of segregation in relation to a social policy such as bringing about school integration where $\mathrm{D}$ could be seen as superior to S in signaling how much potential "social disruption" will be involved in achieving segregation. This would be sociologically important regardless of whether movement to achieve integration brings about big changes in racial proportions in different schools.

At the same time, I argue against the prevailing view that G, D, and R should be seen as the best available choices or even appropriate choices for serving most research interests. Personally, I am interested in measures of uneven distribution that are well suited for signaling the consequences segregation may have for group differences in residential outcomes that are both meaningful to individuals and households and relevant for life chances associated with residential outcomes. Given this focus, I am drawn to $\mathrm{S}$ because, among popular indices of uneven distribution, it registers residential outcomes that have clear and compelling implications for racial differences in residential outcomes. Focusing on the example of WhiteBlack segregation, I know that when $\mathrm{S}$ is high, Whites and Blacks are residentially separated and are living apart from each other in neighborhoods that differ markedly on racial mix. I also know that there is a clear structural potential for the 
neighborhoods that Whites and Blacks live in to differ in other respects as well (e.g., amenities, crime, poverty, exposure to social problems, etc.). Furthermore, I know that when $\mathrm{S}$ is high, $\mathrm{R}, \mathrm{G}$, and D also will be high and as a result knowledge of their values adds limited additional information that is relevant to my concerns.

When $\mathrm{S}$ is low, I know that Whites and Blacks are not residentially separated; instead, they are living together in the same neighborhoods. Because of this, I additionally, know that, all else equal, the possibility for Whites and Blacks to experience fundamentally different neighborhood outcomes on other dimensions (e.g., amenities, crime, poverty, etc.) is logically constrained because people who reside in the same neighborhoods necessarily experience the same neighborhood outcomes. If $\mathrm{S}$ is exactly zero, Whites and Blacks cannot on average experience different neighborhood outcomes based on race alone. As $S$ takes higher values, the logical potential increases for Whites and Blacks to differ on residential outcomes based on race alone.

Of course $\mathrm{S}$ does not reflect all relevant aspects of race differences in residential outcomes by itself. Other characteristics such as income can interact with race and influence race differences in neighborhood outcomes. For example, a low-tomoderate level of S, say 15-20, could result because Whites and Blacks have substantial contact across all income strata. Alternatively, the same level of S may result due to Blacks having higher levels of contact with low income Whites that offset Blacks having lower levels of contact with high income Whites. All else equal, the second scenario will be associated with greater White-Black differences in exposure to poverty and low income. This does not change the fundamental implications of high $\mathrm{S}$ versus low $\mathrm{S}$ situations. It merely acknowledges that consequences of racial differences in residential distributions are not necessarily simple.

What can be said about White-Black neighborhood differences when R, G, and $\mathrm{D}$ take high values? This is much harder to pin down. When $\mathrm{S}$ is high, $\mathrm{R}, \mathrm{G}$, and D will be high. But the reverse is not true. S can be low when R, G, and D are high, particularly when group size is highly imbalanced. When this occurs, the high values of $\mathrm{R}, \mathrm{G}$, and $\mathrm{D}$ do not provide a basis on their own for offering conclusions regarding White-Black differences in residential outcomes. This monograph has established that, as a matter of arithmetic, when R, G, and D have high values when $\mathrm{S}$ is low it is because these indices are responding strongly to small quantitative differences on neighborhood racial mix (p) over relatively narrow ranges of $p$. This provides little basis for speculating about the consequences of uneven distribution for residential differences. This is made worse by the fact the "crucial" range of $p$ varies from city-to-city depending on racial mix. For my research interests, this index behavior is not attractive.

What about Theil's H which I have not yet discussed? Like S, Theil's H usually receives favorable treatment in methodological studies of segregation indices but has been used less frequently than D in empirical studies. For purposes of this discussion, $\mathrm{H}$ falls between $\mathrm{S}$ and indices rooted in the segregation curve $(\mathrm{G}, \mathrm{D}$, and $\mathrm{R})$. Figures 6.5 and 6.6 introduced earlier show that the function $y=f(p)$ for $H$ is similar to the same function for $\mathrm{R}$ in several respects. The nonlinearity in the $\mathrm{y}-\mathrm{p}$ relationship is similar in form, but the magnitude of the departure from nonlinearity is much less 

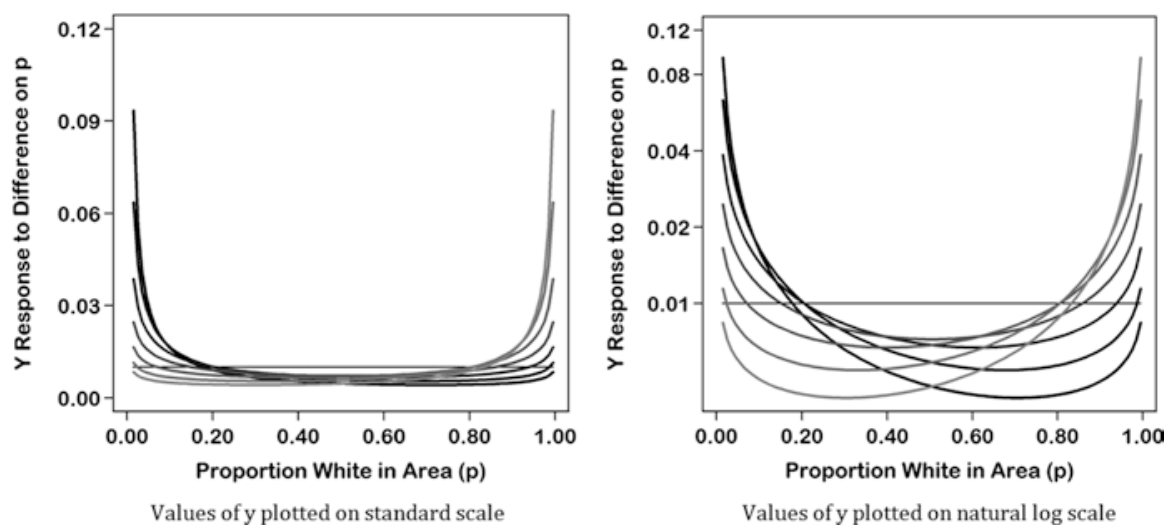

Fig. 12.2 Response of group contact (y) scored for Theil's $\mathrm{H}$ by proportion White in area (p) and selected values for city proportion White (P) (Curves reflect the response of Theil's $\mathrm{H}$ to a change in area proportion White (p) by the level of $\mathrm{p}$ and selected values of proportion White for the city $(P) . y=f(p)=Q+[(E-e) / E] /(p / P-q / Q)$. Moving from darker curves to lighter curves, the values of $P$ are: $0.01,0.05,0.20,0.50,0.80,0.95$, and 0.99 . The horizontal line is for reference and reflects the "flat" response of the separation index $(\mathrm{S})$ )

dramatic and the degree to which it varies with city racial mix (P) also is less dramatic. So, in comparison with $\mathrm{S}, \mathrm{H}$ has similar tendencies as $\mathrm{R}$, but in milder degree. Figures 12.1 and 12.2 document that $\mathrm{H}$ has similar tendencies to $\mathrm{R}$ in terms of how changes in area racial composition (p) translate into changes in residential outcomes (y). The figures document similarity in the form of the response. One must note the values on the "Y" scale in the figures to see that the responses by $\mathrm{H}$ are milder than the responses by $\mathrm{R}$.

What distinguishes $\mathrm{H}$ from $\mathrm{R}$ is this. $\mathrm{H}$ is rooted in a conception of uneven distribution that draws on the information-theoretic notion of relative deviation from expected entropy. Individuals who find this conceptual approach attractive may accordingly prefer $\mathrm{H}$. But like $\mathrm{G}, \mathrm{D}$, and $\mathrm{R}, \mathrm{H}$ is differentially sensitive to changes in $p$ over relatively narrow ranges and the relevant ranges vary with city racial mix. I am not aware of a basis for prizing this quality and leave it for others to make the case.

\subsection{An Example Analysis of Segregation and Exposure to Neighborhood Poverty}

I conclude this chapter by presenting an empirical analysis intended to speak to the issues reviewed here in a more "concrete" way. The issue I explore is whether high scores for measures of uneven distribution carry implications for racial stratification on residential and neighborhood outcomes. To investigate this, I used block group data from Summary File 3 of the 2000 census and computed scores for the indices 
of uneven distribution discussed in this section - specifically, G, D, R, H, and S - for Core Based Statistical Areas (CBSAs). I computed scores for White-Minority comparisons - specifically, White-Black, White-Latino, and White-Asian - using data for non-Hispanics for Whites, Blacks, and Asians. For economy of presentation, I focus on the results for $\mathrm{D}$ and $\mathrm{S}$, noting that index scores for $\mathrm{G}$ and $\mathrm{R}$ correlate closely with scores for $\mathrm{D}$ and noting that scores for $\mathrm{H}$ takes an intermediate position between scores for $\mathrm{D}$ and $\mathrm{S}$.

I additionally calculated group-specific exposure to neighborhood poverty based on poverty rates for neighborhoods (calculated using data for the total population) and also group-specific exposure to neighborhood income rank (percentile standing based on the city-specific income distribution for the total population). I then calculated the White-Black, White-Latino, and White-Asian differences on exposure to neighborhood poverty and exposure to neighborhood income rank. The differences were constructed so positive scores indicated White advantage. ${ }^{2}$ I restricted the analysis to CBSAs where the minority group in the segregation comparison had a population of 1,500 or more and where the number of block-groups was adequate for assessing segregation patterns. ${ }^{3}$ This resulted in 1,455 CBSA-group comparisons; 571 White-Black comparisons, 605 White-Latino comparisons, and 279 White-Asian comparisons.

I then addressed the following question; "Do scores on D and S for WhiteMinority segregation carry similar or different implications for White-Minority differences on these residential inequality outcomes?" To a certain extent they do carry similar implications, at least in this analysis, as the scores for both $\mathrm{D}$ and $\mathrm{S}$ are positively associated with White-Minority inequality on exposure to poverty and neighborhood income rank. The White-Minority difference in exposure to neighborhood poverty (coded so higher scores indicate White advantage) is correlated with $\mathrm{D}$ at $0.645\left(\mathrm{r}^{2}=0.417\right)$ and with $\mathrm{S}$ at $0.715\left(\mathrm{r}^{2}=0.512\right)$. The White-Minority difference in exposure to neighborhood income rank (also coded so higher scores indicate White advantage $)$ is correlated with $\mathrm{D}$ at $0.619\left(\mathrm{r}^{2}=0.383\right)$ and with $\mathrm{S}$ at 0.702 $\left(r^{2}=0.494\right)$. These results indicate that $\mathrm{S}$ provides a better signal for when segregation carries implications for racial inequality in neighborhood outcomes. But in this analysis D is not awful for this purpose. One reason for this is that scores on D and $\mathrm{S}$ are often concordant. The story changes substantially when attention is focused on cases where $\mathrm{D}$ and $\mathrm{S}$ are discordant.

Probing more deeply into the data lends additional support to the idea that $\mathrm{S}$ is more attractive than D for the purpose of signaling when it is likely that segregation is associated with White-Minority inequality in residential outcomes. To do this, I coded each White-Minority segregation comparison on the consistency of D and

\footnotetext{
${ }^{2}$ The poverty difference is minority exposure minus White exposure. Positive scores indicate that the minority group is exposed to higher levels of neighborhood poverty than White. The income rank difference is White exposure minus minority exposure with positive scores indicating Whites are exposed to neighborhoods that rank higher on income (White advantage).

${ }^{3}$ The cut-off was at least 10 populated block groups. I replicated the results using cut-off values of 15 and 20 block groups. The results were the same.
} 
S. Recall that D can take high values when $S$ is low. Based on this, I classified outcomes on D and S into four categories. The first is a baseline category of "concordant" as occurs in prototypical segregation where displacement from even distribution is substantially polarized. The other three categories capture D exceeding $\mathrm{S}$ by increasingly large amounts. Holding $\mathrm{D}$ constant, distribution across the three categories of D-S discrepancy indicates variation in the extent to which displacement from uneven distribution is dispersed and produces lower levels of group separation and neighborhood polarization.

I then estimated the regression of the White-Minority difference on exposure to neighborhood poverty on D and the three categories of D-S discrepancy. The multiple R-square for the regression was 0.502 compared to 0.417 when using $\mathrm{D}$ alone. This indicates that knowing that $\mathrm{D}$ is discordant from $\mathrm{S}$ added to the ability to predict the White-Minority difference in exposure to neighborhood poverty over what could be predicted from knowledge of $\mathrm{D}$ alone. As expected, the pattern of the effects indicated that when $\mathrm{D}$ was high in relation to $\mathrm{S}$, the White-Minority difference in exposure to neighborhood poverty was lower (all effects were statistically significant at $\mathrm{p}<0.001)$. The impact of the largest $\mathrm{D}-\mathrm{S}$ discrepancy category was -4.3 which is clearly large in relation to the value of 6.9 for interquartile range of 6.9 for the dependent variable.

I obtained similar results for the regression predicting the White-Minority difference on neighborhood income rank. The multiple R-square for the regression using $\mathrm{D}$ and the three categories of D-S discrepancy as predictors was 0.483 compared to 0.383 when using $\mathrm{D}$ alone. The results indicated that knowing that $\mathrm{D}$ was high in relation to $\mathrm{S}$ added to the ability to predict White-Minority difference in exposure to neighborhood income rank over what could be predicted from knowledge of $\mathrm{D}$ alone (all effects statistically significant at $\mathrm{p}<0.001$ ). As expected, discrepant categories had lower levels of White-Black inequality on income rank and the impact of the largest D-S discrepancy category was -4.0 which is clearly large when compared to the value of the interquartile range of 5.8 for the dependent variable.

I next estimated parallel regressions where $\mathrm{S}$ and categories of D-S discrepancy were used to predict White-Minority disadvantage in exposure to poverty and neighborhood income rank. The results were different and quite revealing. For the regression of the White-Minority difference on exposure to neighborhood poverty the multiple R-square for the regression was 0.529 compared to 0.512 when using $\mathrm{S}$ alone. This signals that knowing $\mathrm{D}$ was high relative to $\mathrm{S}$ increased the ability to predict the White-Minority difference in exposure to neighborhood poverty by only a small amount over what could be predicted from knowledge of $\mathrm{S}$ alone. The coefficients for the three categories of discrepancy were all statistically significant (all at $\mathrm{p}<0.001$ ) but impacts were more modest than in the parallel analysis focusing on $\mathrm{D}$ as the largest effect here was 1.9 which was less than half the size of the largest effect of -4.3 seen in the parallel analysis focusing on D.

I found similar results for the regression predicting the White-Minority difference on neighborhood income rank. The multiple R-square for the regression using $\mathrm{S}$ and the three categories of D-S discrepancy as predictors was 0.508 compared to 0.494 
when using $\mathrm{S}$ alone. So, again, knowing that $\mathrm{D}$ was high relative to $\mathrm{S}$ increased ability to predict White-Minority difference in exposure to neighborhood income rank by only a small amount over what could be achieved from knowledge of $\mathrm{S}$ alone. The effect coefficients for D-S discrepancy were statistically significant (all at $\mathrm{p}<0.001$ ), but effects were small compared to the parallel analysis focusing on $\mathrm{D}$ as the largest effect was 1.5 compared to -4.0 in the parallel analysis focusing on D.

I draw the following conclusions based on these analyses. In comparison with the dissimilarity index $(D)$, the separation index $(S)$ speaks more directly to the question of whether uneven distribution is associated with group differences in residential outcomes such as income and poverty. This is because $\mathrm{S}$ registers whether or not groups live separately in neighborhoods that are polarized on racial mix. This is a logical precondition for White-Minority differences on neighborhood-level stratification outcomes such as socioeconomic standing. D can take high values when groups live together in neighborhoods with similar racial composition and the logical potential for group differences in neighborhood outcomes is limited. Accordingly, $\mathrm{S}$ is the stronger predictor of White-Minority differences on neighborhood-based stratification outcomes such as indicators of neighborhood socioeconomic standing. Not surprisingly, I obtained parallel findings when contrasting $\mathrm{S}$ with the gini index $(\mathrm{G})$ and the Hutchens square root index $(\mathrm{R})$. This is because these two measures correlate closely with $\mathrm{D}$ and can take high values when groups are not residentially separated.

In view of these results, I suggest that researchers always examine multiple indices and give particularly close attention to cases where $\mathrm{S}$ and $\mathrm{D}$ (or its close correlates) diverge. Such cases involve uneven distribution without residential separation and neighborhood polarization. These situations are likely to be fundamentally different from cases of prototypical segregation where $\mathrm{D}$ and $\mathrm{S}$ both take high values. Specifically, group inequality in neighborhood-based residential outcomes is likely to be higher under a high level of prototypical segregation (i.e., a high-D, high-S combination) and lower under a high level of "displacement without separation" (i.e., a high-D, low-S combination). Personally, I am primarily interested in those aspects of segregation that have greater potential consequences for stratification in neighborhood outcomes and associated life chances. So I pay closer attention to $\mathrm{S}$ when $\mathrm{S}$ and $\mathrm{D}$ disagree.

Open Access This chapter is licensed under the terms of the Creative Commons AttributionNonCommercial 2.5 International License (http://creativecommons.org/licenses/by-nc/2.5/), which permits any noncommercial use, sharing, adaptation, distribution and reproduction in any medium or format, as long as you give appropriate credit to the original author(s) and the source, provide a link to the Creative Commons license and indicate if changes were made.

The images or other third party material in this chapter are included in the chapter's Creative Commons license, unless indicated otherwise in a credit line to the material. If material is not included in the chapter's Creative Commons license and your intended use is not permitted by statutory regulation or exceeds the permitted use, you will need to obtain permission directly from the copyright holder.

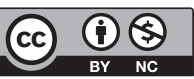

